UCRHEP-T124

April 1994

\title{
Left-Right Symmetry and Supersymmetric Unification
}

\author{
Ernest Ma \\ Department of Physics \\ University of California \\ Riverside, California 92521
}

\begin{abstract}
The existence of an $\mathrm{SU}(3) \times \mathrm{SU}(2)_{\mathrm{L}} \times \mathrm{SU}(2)_{\mathrm{R}} \times \mathrm{U}(1)$ gauge symmetry with $g_{\mathrm{L}}=g_{\mathrm{R}}$ at the $\mathrm{TeV}$ energy scale is shown to be consistent with supersymmetric $\mathrm{SO}(10)$ grand unification at around $10^{16} \mathrm{GeV}$ if certain new particles are assumed. The additional imposition of a discrete $\mathrm{Z}_{2}$ symmetry leads to a generalized definition of $\mathrm{R}$ parity as well as highly suppressed Majorana neutrino masses. Another model based on $\mathrm{SO}(10) \times \mathrm{SO}(10)$ is also discussed.
\end{abstract}


Possible unification of the three gauge couplings of the standard $\mathrm{SU}(3)_{\mathrm{C}} \times \mathrm{SU}(2)_{\mathrm{L}} \times \mathrm{U}(1)_{\mathrm{Y}}$ model of quark and lepton interactions in its minimal supersymmetric extension has revived widespread interest in this topic. [1] The success of such a scenario seems to imply that no new physics beyond the minimal supersymmetric standard model (MSSM) would be observed below an energy scale of a few $\mathrm{TeV}$. In particular, the left-right gauge extension appears to be excluded, except for one very special case, 2] but even then, the condition $g_{\mathrm{L}}=g_{\mathrm{R}}$ cannot be maintained.[3] If $g_{\mathrm{L}}=g_{\mathrm{R}}$ is desired, the scale $\mathrm{M}_{\mathrm{R}}$ of $\mathrm{SU}(2)_{\mathrm{R}}$ symmetry breaking obtained in previous studies [4] is typically of the order $10^{10} \mathrm{GeV}$. The purpose of this paper is to show in an explicit first example that it is actually possible to have supersymmetric $\mathrm{SO}(10)$ grand unification at around $10^{16} \mathrm{GeV}$ as well as an $\mathrm{SU}(3) \times \mathrm{SU}(2)_{\mathrm{L}} \times \mathrm{SU}(2)_{\mathrm{R}} \times \mathrm{U}(1)$ gauge symmetry with $g_{\mathrm{L}}=g_{\mathrm{R}}$ at the TeV energy scale. New particles will be assumed together with a discrete $\mathrm{Z}_{2}$ symmetry, resulting in a generalized definition of $\mathrm{R}$ parity together with highly suppressed Majorana neutrino masses. Another model based on $\mathrm{SO}(10) \times \mathrm{SO}(10)$ will also be discussed.

Consider a supersymmetric SO(10) model which breaks down at the unification scale $\mathrm{M}_{\mathrm{U}}$ to a supersymmetric $\mathrm{SU}(3) \times \mathrm{SU}(2)_{\mathrm{L}} \times \mathrm{SU}(2)_{\mathrm{R}} \times \mathrm{U}(1)$ model with left-right exchange symmetry, which then breaks down at $\mathrm{M}_{\mathrm{R}}$ to the standard (nonsupersymmetric) $\mathrm{SU}(3) \times$ $\mathrm{SU}(2)_{\mathrm{L}} \times \mathrm{U}(1)_{\mathrm{Y}}$ model. The scale of supersymmetry breaking is assumed to coincide with $M_{R}$. Before going into the details of the evolution of the various gauge couplings, note that with a left-right model, there should be two scalar bidoublets in order that realistic quark and lepton mass matrices be obtained. Hence there are four SU(2) L doublets which will change the evolution of the $\mathrm{SU}(2)_{\mathrm{L}}$ coupling $g_{\mathrm{L}}$ but not that of the $\mathrm{SU}(3)$ coupling $g_{\mathrm{S}}$, leading to the loss of unification of the gauge couplings. In the MSSM, this corresponds to the well-known fact that unification occurs with two, but not four, Higgs doublets. Note also that this problem exists whether or not $g_{\mathrm{L}}=g_{\mathrm{R}}$ unless $\mathrm{M}_{\mathrm{R}}=\mathrm{M}_{\mathrm{U}}$. Consequently, new 
particles are necessary to offset the effect of the extra bidoublet if $\mathrm{SU}(2)_{\mathrm{R}}$ is to be a gauge symmetry already above a few $\mathrm{TeV}$.

Consider now the evolution of the gauge couplings to one-loop order. Generically,

$$
\alpha_{i}^{-1}\left(M_{1}\right)=\alpha_{i}^{-1}\left(M_{2}\right)-\frac{b_{i}}{2 \pi} \ln \frac{M_{1}}{M_{2}}
$$

where $\alpha_{i} \equiv g_{i}^{2} / 4 \pi$ and $b_{i}$ are constants determined by the particle content contributing to $\alpha_{i}$. The initial conditions are set at $\mathrm{M}_{\mathrm{Z}}=91.187 \pm 0.007 \mathrm{GeV}[5]$ by the experimental values $\alpha^{-1}=127.9 \pm 0.1,\left[\right.$ [6] $\sin ^{2} \theta_{\mathrm{W}}=0.2321 \pm 0.0006$, [7] and $\alpha_{\mathrm{S}}=0.120 \pm 0.006 \pm 0.002$. [8] Hence

$$
\begin{gathered}
\alpha_{\mathrm{S}}^{-1}\left(\mathrm{M}_{\mathrm{Z}}\right)=8.33^{+0.60}-0.52 \\
\alpha_{\mathrm{L}}^{-1}\left(\mathrm{M}_{\mathrm{Z}}\right)=29.69 \pm 0.10
\end{gathered}
$$

and

$$
\alpha_{\mathrm{Y}}^{-1}\left(\mathrm{M}_{\mathrm{Z}}\right)=98.21 \pm 0.15
$$

At $\mathrm{M}_{\mathrm{R}}$, the matching conditions of the gauge couplings are

$$
\alpha_{\mathrm{L}}^{-1}\left(\mathrm{M}_{\mathrm{R}}\right)=\alpha_{\mathrm{R}}^{-1}\left(\mathrm{M}_{\mathrm{R}}\right),
$$

and

$$
\alpha_{\mathrm{Y}}^{-1}\left(\mathrm{M}_{\mathrm{R}}\right)=\alpha_{\mathrm{R}}^{-1}\left(\mathrm{M}_{\mathrm{R}}\right)+\alpha_{\mathrm{X}}^{-1}\left(\mathrm{M}_{\mathrm{R}}\right),
$$

where $\alpha_{\mathrm{X}}$ refers to the $\mathrm{U}(1)$ gauge coupling of the left-right symmetry. Above $\mathrm{M}_{\mathrm{R}}, \alpha_{\mathrm{L}}$ and $\alpha_{\mathrm{R}}$ will evolve together identically.

The particle content of this model is as follows. There are three copies of the $\mathbf{1 6}$ representation of $\mathrm{SO}(10)$ consisting of the usual quarks and leptons. Their transformations under $\mathrm{SU}(3) \times \mathrm{SU}(2)_{\mathrm{L}} \times \mathrm{SU}(2)_{\mathrm{R}} \times \mathrm{U}(1)$ are given by

$$
Q \sim(3,2,1,1 / 6), \quad Q^{c} \sim(\overline{3}, 1,2,-1 / 6),
$$


and

$$
L \sim(1,2,1,-1 / 2), \quad L^{c} \sim(1,1,2,1 / 2)
$$

There are two bidoublets

$$
\Phi_{12} \sim(1,2,2,0)
$$

which are necessary for realistic quark and lepton mass matrices as already mentioned, and one set of $\mathrm{SU}(2)_{\mathrm{L}}$ and $\mathrm{SU}(2)_{\mathrm{R}}$ doublets and their charge-conjugate partners

$$
\Phi_{\mathrm{L}} \sim(1,2,1,-1 / 2), \quad \Phi_{\mathrm{R}} \sim(1,1,2,1 / 2)
$$

and

$$
\Phi_{\mathrm{L}}^{c} \sim(1,2,1,1 / 2), \quad \Phi_{\mathrm{R}}^{c} \sim(1,1,2,-1 / 2)
$$

so that $\mathrm{SU}(2)_{\mathrm{R}}$ may be broken independently of $\mathrm{SU}(2)_{\mathrm{L}}$. Added to this minimal collection are two copies each of

$$
D \sim(3,1,1,-1 / 3), \quad D^{c} \sim(\overline{3}, 1,1,1 / 3)
$$

and

$$
E \sim(1,1,1,-1), \quad E^{c} \sim(1,1,1,1)
$$

as well as three copies of

$$
N \sim(1,1,1,0)
$$

As shown below, this choice will allow $\mathrm{M}_{\mathrm{R}}$ to be a few $\mathrm{TeV}$ with $\mathrm{M}_{\mathrm{U}}$ of the order $10^{16} \mathrm{GeV}$.

In the one-loop approximation, below $\mathrm{M}_{\mathrm{R}}$,

$$
\begin{aligned}
b_{\mathrm{S}} & =-11+\frac{4}{3}(3)=-7, \\
b_{\mathrm{L}} & =-\frac{22}{3}+\frac{4}{3}(3)+\frac{1}{6}(2)=-3, \\
b_{\mathrm{Y}} & =\frac{20}{9}(3)+\frac{1}{6}(2)=7,
\end{aligned}
$$


whereas above $\mathrm{M}_{\mathrm{R}}$,

$$
\begin{aligned}
b_{\mathrm{S}} & =-9+2(3)+n_{D}=-1, \\
b_{\mathrm{LR}} & =-6+2(3)+n_{22}+n_{H}=3, \\
\frac{3}{2} b_{\mathrm{X}} & =2(3)+3 n_{H}+n_{D}+3 n_{E}=17,
\end{aligned}
$$

where $n_{22}=2, n_{H}=1, n_{D}=2, n_{E}=2$, and the factor $3 / 2$ for $b_{\mathrm{X}}$ comes from the normalization of the $\mathrm{U}(1)_{\mathrm{X}}$ coupling within $\mathrm{SO}(10)$. Assuming that $\alpha_{\mathrm{S}}^{-1}\left(\mathrm{M}_{\mathrm{U}}\right)=\alpha_{\mathrm{LR}}^{-1}\left(\mathrm{M}_{\mathrm{U}}\right)=$ $(3 / 2) \alpha_{\mathrm{X}}^{-1}\left(\mathrm{M}_{\mathrm{U}}\right)$, Eq. (1) can be solved for $\mathrm{M}_{\mathrm{R}}$ and $\mathrm{M}_{\mathrm{U}}$, i.e.

$$
\ln \frac{\mathrm{M}_{\mathrm{R}}}{\mathrm{M}_{\mathrm{Z}}}=\frac{\pi}{4}\left[3 \alpha^{-1}\left(\mathrm{M}_{\mathrm{Z}}\right)\left\{1-5 \sin ^{2} \theta_{\mathrm{W}}\left(\mathrm{M}_{\mathrm{Z}}\right)\right\}+7 \alpha_{\mathrm{S}}^{-1}\left(\mathrm{M}_{\mathrm{Z}}\right)\right]<1.66
$$

and

$$
\ln \frac{\mathrm{M}_{\mathrm{U}}}{\mathrm{M}_{\mathrm{Z}}}=\frac{\pi}{2}\left[\alpha^{-1}\left(\mathrm{M}_{\mathrm{Z}}\right) \sin ^{2} \theta_{\mathrm{W}}\left(\mathrm{M}_{\mathrm{Z}}\right)-\alpha_{\mathrm{S}}^{-1}\left(\mathrm{M}_{\mathrm{Z}}\right)\right]>32.45 .
$$

Hence $\mathrm{M}_{\mathrm{R}}<480 \mathrm{GeV}$ and $\mathrm{M}_{\mathrm{U}}>1.1 \times 10^{16} \mathrm{GeV}$. The upper bound of $\mathrm{M}_{\mathrm{U}}$ is $1.9 \times 10^{16} \mathrm{GeV}$, corresponding to $\mathrm{M}_{\mathrm{R}}=\mathrm{M}_{\mathrm{Z}}$.

The allowed parameter space opens up more in two loops. Using [9]

$$
b_{i j}=\left(\begin{array}{ccc}
-26 & \frac{9}{2} & \frac{11}{10} \\
12 & 8 & \frac{6}{5} \\
\frac{44}{5} & \frac{18}{5} & \frac{104}{25}
\end{array}\right)
$$

for $\alpha_{\mathrm{S}}^{-1}, \alpha_{\mathrm{L}}^{-1}$, and $(3 / 5) \alpha_{\mathrm{Y}}^{-1}$ below $\mathrm{M}_{\mathrm{R}}$, and

$$
b_{i j}=\left(\begin{array}{ccc}
\frac{110}{3} & 9 & \frac{7}{3} \\
24 & 45 & \frac{9}{2} \\
\frac{56}{3} & \frac{27}{2} & \frac{293}{6}
\end{array}\right)
$$

for $\alpha_{\mathrm{S}}^{-1}, \alpha_{\mathrm{LR}}^{-1}$, and $(3 / 2) \alpha_{\mathrm{X}}^{-1}$ above $\mathrm{M}_{\mathrm{R}}$, and solving the equations

$$
\mu \frac{\partial \alpha_{i}(\mu)}{\partial \mu}=\frac{1}{2 \pi}\left(b_{i}+\frac{b_{i j}}{4 \pi} \alpha_{j}(\mu)\right) \alpha_{i}^{2}(\mu)
$$

numerically with the proper boundary conditions at $\mathrm{M}_{\mathrm{U}}$ :

$$
\alpha_{\mathrm{U}}^{-1}-\frac{2}{3 \pi}=\alpha_{\mathrm{S}}^{-1}-\frac{1}{4 \pi}=\alpha_{\mathrm{LR}}^{-1}-\frac{1}{6 \pi}=\frac{3}{2} \alpha_{\mathrm{X}}^{-1},
$$


it is found that

$$
6.3 \times 10^{15} \mathrm{GeV}<\mathrm{M}_{\mathrm{U}}<2.3 \times 10^{16} \mathrm{GeV}
$$

with

$$
\text { 6.7 } \mathrm{TeV}>\mathrm{M}_{\mathrm{R}}>\mathrm{M}_{\mathrm{Z}}
$$

As an example, Fig. 1 shows the case where $M_{R}$ is chosen arbitrarily to be 1 TeV with the central values $\alpha^{-1}\left(\mathrm{M}_{\mathrm{Z}}\right)=127.9$ and $\sin ^{2} \theta_{\mathrm{W}}\left(\mathrm{M}_{\mathrm{Z}}\right)=0.2321$ as inputs, from which $\mathrm{M}_{\mathrm{U}}=$ $1.0 \times 10^{16} \mathrm{GeV}$ and $\alpha_{\mathrm{S}}\left(\mathrm{M}_{\mathrm{Z}}\right)=0.115$ are obtained. Note that $\mathrm{M}_{\mathrm{R}}$ depends very sensitively on $\alpha_{\mathrm{S}}$. If $\alpha_{\mathrm{S}}\left(\mathrm{M}_{\mathrm{Z}}\right)=0.120$ is used in the above, $\mathrm{M}_{\mathrm{R}}$ drops down by an order of magnitude to $120 \mathrm{GeV}$, whereas $\mathrm{M}_{\mathrm{U}}$ increases only about twofold to $1.8 \times 10^{16} \mathrm{GeV}$.

The matter superfields of this model are assumed to be distinguished by a discrete $\mathrm{Z}_{2}$ symmetry where $\Phi_{\mathrm{R}}, \Phi_{\mathrm{R}}^{c}$, and $\Phi_{12}$ are even, and all the others are odd. This is merely a generalization of the usual procedure in the MSSM where the two Higgs superfields are chosen to be even, and the quark and lepton superfields odd. As a result, a generalized conserved $\mathrm{R}$ parity also exists in this model. Note that the terms $Q Q D, Q^{c} Q^{c} D^{c}, L Q D^{c}$, and $L^{c} Q^{c} D$ are all forbidden.

The spontaneous breaking of the $\mathrm{SU}(2)_{\mathrm{R}} \times \mathrm{U}(1)_{\mathrm{X}}$ gauge symmetry down to $\mathrm{U}(1)_{\mathrm{Y}}$ is accomplished by the nonzero vacuum expectation values of the neutral scalar components of $\Phi_{\mathrm{R}}$ and $\Phi_{\mathrm{R}}^{c}$. Similarly, $\mathrm{SU}(2)_{\mathrm{L}} \times \mathrm{U}(1)_{\mathrm{Y}}$ breaks down to $\mathrm{U}(1)_{\mathrm{Q}}$ via $\Phi_{12}$. The usual quarks and leptons obtain their masses through the Yukawa terms $Q Q^{c} \Phi_{12}$ and $L L^{c} \Phi_{12}$. The exotic quarks and leptons have gauge-invariant mass terms $D D^{c}$ and $E E^{c}$. Mixing between the two sectors occurs through the terms $D Q^{c} \Phi_{\mathrm{R}}$ and $E L^{c} \Phi_{\mathrm{R}}$. This means that whereas $d^{c}-D^{c}$ and $e^{c}-E^{c}$ mixing may be substantial, $d-D$ and $e-E$ mixing are guaranteed to be small. [10] Although $\Phi_{\mathrm{L}}$ and $L$ transform identically and neither have any vacuum expectation value, the gauge-invariant mass term $\Phi_{\mathrm{L}} \Phi_{\mathrm{L}}^{c}$ can be used to define $\Phi_{\mathrm{L}}$, after which there is of course still an allowed $\Phi_{\mathrm{L}} L^{c} \Phi_{12}$ term, but the $L-\Phi_{\mathrm{L}}$ mixing will be highly suppressed. 10] 
Consider now the neutrino sector. Ignoring the small mixing with the neutral spinor components of $\Phi_{\mathrm{L}}$ and $\Phi_{\mathrm{L}}^{c}$, the mass matrix spanning $\nu, \nu^{c}$, and $N$ is of the form

$$
\mathcal{M}=\left(\begin{array}{ccc}
0 & m_{\mathrm{D}} & 0 \\
m_{\mathrm{D}} & 0 & m_{\mathrm{R}} \\
0 & m_{\mathrm{R}} & m_{N}
\end{array}\right)
$$

where $m_{\mathrm{D}}$ comes from the $L L^{c} \Phi_{12}$ term, $m_{\mathrm{R}}$ from the $N L^{c} \Phi_{\mathrm{R}}^{c}$ term, and $m_{N}$ is an allowed gauge-invariant Majorana mass term for $N$. Note that if $m_{N}=0$, then additive lepton number is conserved and the matrix $\mathcal{M}$ has a zero eigenvalue which is the physical mass of a linear combination of $\nu$ and $N$, while its orthogonal combination pairs up with $\nu^{c}$ to form a heavy Dirac fermion of mass $\sqrt{m_{\mathrm{R}}^{2}+m_{\mathrm{D}}^{2}}$. If $m_{N}$ is not zero but nevertheless small, $\nu$ will pick up a very small Majorana mass given by

$$
m_{\nu} \simeq \frac{m_{N} m_{\mathrm{D}}^{2}}{m_{\mathrm{R}}^{2}} .
$$

Hence $m_{\nu}$ is not only suppressed by the usual seesaw mechanism, but also by the small ratio $m_{N} / m_{\mathrm{R}}$. 111 This allows $m_{\nu}$ to be very small even though $\mathrm{M}_{\mathrm{R}}$ is only a few TeV. For example, if $m_{\mathrm{D}}=1 \mathrm{MeV}, \mathrm{M}_{\mathrm{R}}=1 \mathrm{TeV}$, and $m_{N}=1 \mathrm{GeV}$, then $m_{\nu} \simeq 10^{-3} \mathrm{eV}$. Note that if $\mathcal{M}$ has a $\nu N$ mass term $m_{\mathrm{L}}$, then $m_{\nu}$ has an additional contribution $\simeq-2 m_{\mathrm{D}} m_{\mathrm{L}} / m_{\mathrm{R}}$ which is not suppressed by $m_{N} / m_{\mathrm{R}}$. However, $m_{\mathrm{L}}$ is absent because the $N L \Phi_{\mathrm{L}}^{c}$ term is forbidden by the assumed discrete $\mathrm{Z}_{2}$ symmetry. On the other hand, left-right exchange symmetry is now broken in the Yukawa sector and the equality $g_{\mathrm{L}}=g_{\mathrm{R}}$ is violated slightly due to the Yukawa contributions to the renormalization-group equations of the gauge couplings, which first appear in two loops.

Consider next an $\mathrm{SO}(10) \times \mathrm{SO}(10)$ model which also breaks down at $\mathrm{M}_{\mathrm{U}}$ to $\mathrm{SU}(3) \times$ $\mathrm{SU}(2)_{\mathrm{L}} \times \mathrm{SU}(2)_{\mathrm{R}} \times \mathrm{U}(1)_{\mathrm{X}}$ but the $\mathrm{U}(1)_{\mathrm{X}}$ is now a linear combination of the usual $\mathrm{U}(1)$ in the first $\mathrm{SO}(10)$ and an $\mathrm{U}(1)$ remnant of the second $\mathrm{SO}(10)$. All low-energy matter supermultiplets are assumed to be only those of the first $\mathrm{SO}(10)$ as well as all gauge particles 
except for the photon (and photino) which spans both $\mathrm{SO}(10)$ 's. Specifically, the electric charge is given by $Q_{1}+Q_{2}$, where $Q_{1}$ and $Q_{2}$ are embedded in the two $\mathrm{SO}(10)$ 's in exactly the same way. The normalization factor for $\alpha_{\mathrm{X}}^{-1}$ is then $3 / 10$ instead of $3 / 2$, because $(3 / 10)^{-1}=$ $(3 / 2)^{-1}+(3 / 8)^{-1}$, assuming of course that the two $\mathrm{SO}(10)$ gauge couplings are equal at $\mathrm{M}_{\mathrm{U}}$.

The particle content of this model is now assumed to consist of three copies of the 16 representation, one copy of the $16^{*}$ representation, two bidoublets, three $N^{\prime}$ 's and one set of $D$ and $D^{c}$. Below $\mathrm{M}_{\mathrm{R}}$, the $b_{i}$ 's are given by $b_{\mathrm{S}}=-11+(4 / 3)(4)=-17 / 3, b_{\mathrm{L}}=$ $-22 / 3+(4 / 3)(4)+(1 / 6)(2)=-5 / 3$, and $b_{\mathrm{Y}}=(20 / 9)(4)+(1 / 6)(2)=83 / 9$. Above $\mathrm{M}_{\mathrm{R}}$, $b_{\mathrm{S}}=-9+2(4)+1=0, b_{\mathrm{LR}}=-6+2(4)+2=4$, and $(3 / 10) b_{\mathrm{X}}=(2 / 5)(4)+1 / 5=9 / 5$. Solving Eq. (1) for $M_{R}$ and $M_{U}$, it is easily seen that $M_{U}$ is again given by Eq. (22) whereas

$$
\ln \frac{\mathrm{M}_{\mathrm{R}}}{\mathrm{M}_{\mathrm{Z}}}=\frac{3 \pi}{37}\left[\frac{3}{2} \alpha^{-1}\left(\mathrm{M}_{\mathrm{Z}}\right)\left\{1-\frac{7}{2} \sin ^{2} \theta_{\mathrm{W}}\left(\mathrm{M}_{\mathrm{Z}}\right)\right\}-\frac{11}{4} \alpha_{\mathrm{S}}^{-1}\left(\mathrm{M}_{\mathrm{Z}}\right)\right]<3.81
$$

Hence $\mathrm{M}_{\mathrm{R}}<4.1 \mathrm{TeV}$ and $\mathrm{M}_{\mathrm{U}}>1.1 \times 10^{16} \mathrm{GeV}$. If the central values of all three experimental inputs are used in the above, $\mathrm{M}_{\mathrm{R}}=2.56 \mathrm{TeV}$ and $\mathrm{M}_{\mathrm{U}}=3.36 \times 10^{16} \mathrm{GeV}$ would be obtained. However, as in the $\mathrm{SO}(10)$ case, two-loop effects are significant and solving Eq. (25) with the appropriate $b_{i j}$ 's, it is found that

$$
\mathrm{M}_{\mathrm{R}}=5.2 \mathrm{TeV}, \quad \mathrm{M}_{\mathrm{U}}=2.0 \times 10^{16} \mathrm{GeV} .
$$

This example is shown in Fig. 2.

A discrete $\mathrm{Z}_{2}$ symmetry is also assumed in this model. The $\mathbf{1 6}^{*}$ and the $\Phi_{12} \sim(1,2,2,0)$ supermultiplets are even, and all the others are odd. The $\mathrm{SU}(2)_{\mathrm{R}} \times \mathrm{U}(1)_{\mathrm{X}}$ gauge symmetry breaks down to $\mathrm{U}(1)_{\mathrm{Y}}$ through the nonzero vacuum expectation value of the $\Phi_{\mathrm{R}}^{c} \sim$ $(1,1,2,-1 / 2)$ component of the $\mathbf{1 6}^{*}$. The breaking of $\mathrm{SU}(2)_{\mathrm{L}} \times \mathrm{U}(1)_{\mathrm{Y}}$ down to $\mathrm{U}(1)_{\mathrm{Q}}$ comes from $\Phi_{12}$ as well as $\Phi_{\mathrm{L}}^{c} \sim(1,2,1,1 / 2)$, but the vacuum expectation value of the latter, which contributes to the mass term $\nu N$, is assumed to be negligibly small in order to have very small Majorana masses for the known neutrinos. As discussed earlier, this term was absent 
in the $\mathrm{SO}(10)$ model because it was possible there to make $\Phi_{\mathrm{L}}^{c}$ odd.

The quarks and leptons within the three $16^{\text {'s }}$ or the $16^{*}$ acquire masses through their couplings with the bidoublets, but the 16 's do not mix with the $16^{*}$ because of the assumed discrete $\mathrm{Z}_{2}$ symmetry. However, they do interact with the three singlet $N$ 's. Hence an exotic $q^{\prime}$ in the $16^{*}$ will decay into its corresponding $q$ in the 16 and a virtual scalar $N$ which then turns into a neutrino and a neutralino which has a $\Phi_{\mathrm{L}}^{c}$ component or a charged lepton and a chargino if kinematically allowed. This differs from the usual models of mirror fermions, [12] i.e. fermions belonging to the $\mathbf{1 6}^{*}$, where they are routinely assumed to mix with the ordinary ones.

In conclusion, it has been shown that it is possible to have an $\mathrm{SU}(3) \times \mathrm{SU}(2)_{\mathrm{L}} \times \mathrm{SU}(2)_{\mathrm{R}} \times$ $\mathrm{U}(1)$ gauge symmetry with $g_{\mathrm{L}}=g_{\mathrm{R}}$ at the TeV energy scale in at least two supersymmetric models of grand unification, based on $\mathrm{SO}(10)$ and $\mathrm{SO}(10) \times \mathrm{SO}(10)$ respectively. New particles are of course necessary but there are simple solutions even though the possibility of unification is very sensitive to small changes in the $b_{i}$ 's. See for example Eqs. (18) to (20). The contributions of the new particles come in large increments, so it is not possible to fine-tune the $b_{i}$ 's to get whatever values of $\mathrm{M}_{\mathrm{U}}$ and $\mathrm{M}_{\mathrm{R}}$ that may be desired. However, as it turns out in both models, the experimental inputs of $\alpha^{-1}, \sin ^{2} \theta_{\mathrm{W}}$, and $\alpha_{\mathrm{S}}$ at $\mathrm{M}_{\mathrm{Z}}$ do happen to require a few $\mathrm{TeV}$ for $\mathrm{M}_{\mathrm{R}}$ and about $10^{16} \mathrm{GeV}$ for $\mathrm{M}_{\mathrm{U}}$. The models are also phenomenologically natural and realistic. Of particular note is the mass matrix of Eq. (29) which results in the highly suppressed Majorana neutrino mass of Eq. (30). New physics at the $\mathrm{TeV}$ energy scale beyond the minimal supersymmetric standard model is clearly possible even in the face of grand unification. 


\section{ACKNOWLEDGEMENT}

I thank T. Yanagida for hospitality at Tohoku University during a recent visit where I had many fruitful discussions with him and other colleagues on the substance of this paper. This work was supported in part by the U. S. Department of Energy under contract No. DE-AT03-87ER40327.

\section{References}

[1] See for example U. Amaldi, W. de Boer, and H. Fürstenau, Phys. Lett. B260, 447 (1991); P. Langacker and M. Luo, Phys. Rev. D44, 817 (1991); J. Ellis, S. Kelley, and D. V. Nanopoulos, Phys. Lett. B287, 95 (1992); V. Barger, M. S. Berger, and P. Ohmann, Phys. Rev. D47, 1093 (1993); M. Carena, S. Pokorski, and C. E. M. Wagner, Nucl. Phys. B406, 59 (1993); and references therein.

[2] N. G. Deshpande, E. Keith, and T. G. Rizzo, Phys. Rev. Lett. 70, 3189 (1993).

[3] D. Chang, R. N. Mohapatra, and M. K. Parida, Phys. Rev. Lett. 52, 1072 (1984).

[4] See for example N. T. Shaban and W. J. Stirling, Phys. Lett. B291, 281 (1992); N. G. Deshpande, E. Keith, and P. B. Pal, Phys. Rev. D46, 2261 (1992); N. G. Deshpande, E. Keith, and P. B. Pal, ibid. 47, 2892 (1993); and references therein.

[5] J. L. Harton, in The Fermilab Meeting (7th Meeting of the American Physical Society Division of Particles and Fields), ed. by C. Albright et al., (World Scientific, Singapore, 1993), p. 397.

[6] G. Degrassi, S. Franchiotti, and A. Sirlin, Nucl. Phys. B351, 49 (1991). 
[7] R. Miquel, in Proc. of 22nd INS International Symposium on Physics with High Energy Colliders (Tokyo, March 1994), to be published.

[8] S. Bethke, in Proc. of 22nd INS International Symposium on Physics with High Energy Colliders (Tokyo, March 1994), to be published.

[9] D. R. T. Jones, Phys. Rev. D25, 581 (1982).

[10] See for example E. Ma, Phys. Lett. B322, 363 (1994).

[11] T. Yanagida, private communication.

[12] For a review, see for example J. Maalampi and M. Roos, Phys. Rep. C186, 53 (1990).

\section{FIGURE CAPTIONS}

Fig. 1. Evolution of $\alpha_{i}^{-1}$ in the $\mathrm{SO}(10)$ model with $\mathrm{M}_{\mathrm{R}}=1 \mathrm{TeV}$ and $\mathrm{M}_{\mathrm{U}}=1.0 \times 10^{16} \mathrm{GeV}$.

Fig. 2. Evolution of $\alpha_{i}^{-1}$ in the $\mathrm{SO}(10) \times \mathrm{SO}(10)$ model with $\mathrm{M}_{\mathrm{R}}=5.2 \mathrm{TeV}$ and $\mathrm{M}_{\mathrm{U}}=$ $2.0 \times 10^{16} \mathrm{GeV}$. 
This figure "fig1-1.png" is available in "png" format from: http://arxiv.org/ps/hep-ph/9405329v1 
This figure "fig1-2.png" is available in "png" format from: http://arxiv.org/ps/hep-ph/9405329v1 